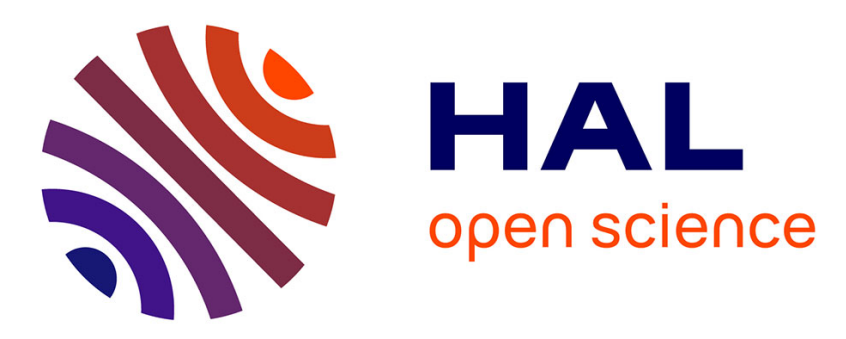

\title{
Higher-order compact scheme for high-performance computing of stratified rotating flows
}

\author{
Stéphane Abide, Stéphane Viazzo, Isabelle Raspo, Anthony \\ Randriamampianina
}

\section{To cite this version:}

Stéphane Abide, Stéphane Viazzo, Isabelle Raspo, Anthony Randriamampianina. Higher-order compact scheme for high-performance computing of stratified rotating flows. Computers and Fluids, 2018, 174, pp.300-310. 10.1016/j.compfluid.2018.07.016 . hal-02111489

\section{HAL Id: hal-02111489 \\ https://hal-amu.archives-ouvertes.fr/hal-02111489}

Submitted on 26 Apr 2019

HAL is a multi-disciplinary open access archive for the deposit and dissemination of scientific research documents, whether they are published or not. The documents may come from teaching and research institutions in France or abroad, or from public or private research centers.
L'archive ouverte pluridisciplinaire HAL, est destinée au dépôt et à la diffusion de documents scientifiques de niveau recherche, publiés ou non, émanant des établissements d'enseignement et de recherche français ou étrangers, des laboratoires publics ou privés. 


\title{
Higher-order compact scheme for high-performance computing of stratified rotating flows
}

\author{
Stéphane Abide ${ }^{\mathrm{a}, *}$, Stéphane Viazzo $^{\mathrm{b}}$, Isabelle Raspo ${ }^{\mathrm{b}}$, Anthony Randriamampianina ${ }^{\mathrm{b}}$ \\ a Université de Perpignan Via Domitia, Perpignan LAMPS EA 4217, France \\ ${ }^{\mathrm{b}}$ Aix Marseille Univ, CNRS, Centrale Marseille, M2P2, Marseille, France
}

\section{A R T I C L E I N F O}

\section{Article history:}

Received 13 April 2018

Revised 5 July 2018

Accepted 23 July 2018

Available online 25 July 2018

\section{Keywords:}

Higher-order compact scheme

High-Performance computing

Rotating stratified flows

2D-pencil decomposition

Reduced parallel diagonal dominant

\begin{abstract}
A B S T R A C T
To take advantage of modern generation computing hardware, a scalable numerical method, based on higher-order compact scheme, is described to solve rotating stratified flows in cylindrical annular domains. An original approach combining 2d-pencil decomposition and reduced Parallel Diagonal Dominant is proposed to improve the parallelization performance during the computation of Poisson/Helmholtz solvers and time explicit terms. The developed technique is validated with respect to analytical solutions, using the method of manufactured solutions, and available data for two specific configurations. The purpose is to demonstrate its ability to correctly capture the flow characteristics in strato-rotational instability and in baroclinic instability with associated small-scale features. Moreover, this code is found to drastically reduce the huge execution times often preventing detailed numerical investigations of these complex phenomena. Strong scaling test is carried out to assess the performance for up to 1024 cores using grid up to $128 \times 568 \times 568$ in radial, axial and azimuthal directions.
\end{abstract}

(c) 2018 Elsevier Ltd. All rights reserved.

\section{Introduction}

Current trends in computational science stem from the increasing prominence of modern multi- and many-core hardware such as graphics processing units (GPUs) in high-performance computing (HPC) infrastructure. Improved multi-core central processing units (CPUs) associated with GPU-accelerated computing can greatly accelerate scientific studies especially in large-scale simulations [1-3]. However, the reduction of execution times strongly depends on the optimization of the performance of discretization methods used on each node while good computational scaling results from small communication footprint [4].

Even though finite element methods are becoming commonplace for simulations of most engineering and biomedical applications on complex geometries, low-order discretizations suffer from strong mesh refinement required to capture certain specific complex solution features. In contrast, high-order techniques, such as spectral approaches, provide improved numerical characteristics at reduced computational cost for a given number of degrees of freedom but they usually remain restricted to regular configurations. Recently, the spectral/hp element methods [5-7] combine high accuracy, known as the spectral convergence, and geometric flex-

\footnotetext{
* Corresponding author.

E-mail address: stephane.abide@univ-perp.fr (S. Abide).
}

ibility to tackle challenging aeronautical flow simulations using between $\mathcal{O}\left(10^{4}\right)$ and $\mathcal{O}\left(10^{5}\right)$ nodes [8], and even up to $\mathcal{O}\left(10^{6}\right)$ nodes [9].

In the present study, a higher-order compact scheme solver [10] is tailored for solving complex rotating stratified flows in annular domains to take advantage of modern generation computing hardware. Our goal is to reduce the huge total time due to the very long transient stage resulting from the thermal stratification associated with the rotation. We have chosen to treat two specific idealized configurations: a Taylor-Couette setup submitted to axial stratification for the strato-rotational instability (SRI) [11] and a uniformly rotating cavity submitted to radial thermal gradient for the baroclinic instability [12]. In the first case, the geometry is defined by a large aspect ratio while the second one is characterized by the coexistence of very different spatio-temporal scales [13]. Before considering more deeply actual physics arising in these two configurations, this work reports on the validation of the developed numerical tool with respect to data available in the literature. We have restricted our simulations to remain in the domain of the Boussinesq approximation: weak density stratification and weak rotation rate to neglect the centrifugal acceleration.

Most of common discretization such as finite volumes, finite differences, finite elements or lattice Boltzmann method, is efficiently implemented in parallel computing environment, even though massively parallel linear solvers still remain a challenging work [14-17]. Concerning high accuracy discretization, the 
picture is more mixed. The computational stencils of such discretizations are larger than those of the common lower-order formulations, therefore inducing large parallel communications. The 2d-pencil decomposition became the common strategy to deal with large computational stencils [18-22]. The successful example of the massively parallel FFT computations with 1d then $2 \mathrm{~d}$ pencil decomposition [21] has been tailoring to the Navier-Stokes solvers based on higher-order discretizations [18,23]. Nowadays, this strategy of parallelization offers a significant reduction of the wall-time and allows the study of highly turbulent flows. Because of the expensive cost of the communications inherent to $2 \mathrm{~d}$ pencil decompositions, some choices in the design of the numerical method must be made to limit communications. For instance, one can choose time-explicit advancement schemes for the diffusive terms, Fourier series expansions, or a collocated variable layout [18]. Compact schemes are finite differences based on implicit relations [24] which rely on solutions of tridiagonal or pentadiagonal linear systems. Therefore, higher-order compact schemes can be considered as an interesting alternative to spectral methods, associating the robustness of finite difference with improved accuracy. In a recent work, Abide et al. [10] proposed an approximate parallel tridiagonal solver [25] to avoid the use of 2d-pencil decomposition for the computation of compact derivatives and interpolations. In this case, the communications between neighbor process rely on halo exchange algorithm. Nevertheless, the 2d-pencil decomposition is still considered to tailor a parallel version of the full diagonalization method [26], despite the cost of the communications involved by the data transpositions. The combination of the two parallelization strategies allows the authors to successfully simulate incompressible flow in truly three-dimensional cartesian domain. Moreover, the basic numerical method features for turbulent flow simulations such as the staggered grid and implicit viscous terms are conserved, while exhibiting a good strong scaling.

The proposed numerical method is based on Fourier/Fourthorder compact scheme discretization defined on staggered grid. The second order Adams-Bashforth/Backward Euler time scheme combined with an efficient projection scheme has been considered: this approach relaxes the stringent time step constraints inherent to the diffusive terms [27]. It corresponds to an extension of a previous numerical code, developed in cartesian coordinates [10], for solving rotating stratified flows in annular domains. Moreover, the present parallelization strategy is concerned with a 2d-pencil decomposition and reduced Parallel Diagonal Dominant (rPDD) to address the numerical solutions of Helmholtz/Poisson problems and the computations of the compact derivatives and interpolations respectively. The implementation of the combination of these two techniques brings novelty and originality to the present approach in comparison with previous works based on higher-order codes [18].

The rotating stratified flows concern a wide spectrum of applications as well as in geophysics such as large scale circulations in atmosphere and oceans, astrophysics such as accretion disk, than in disk storage, turbomachinery ... The Strato-Rotational Instability (SRI) is a purely hydrodynamic instability with distinctive local features and may be studied from specifically designed laboratory experiments and numerical simulations in an axially-stratified Taylor-Couette setup $[11,28,29]$. Here axial stratification is obtained by cooling the bottom and heating the upper lid [11]. The temperature stratification represents the axial stratification of an accretion disk centered around a hot star and the rotation of the cylinders mimics the astrophysical rotation laws with rotation rate decreasing outwards. Understanding the mechanisms that can result in an outward angular momentum transport is the central problem of planet formation, particularly in the theory of accretion disks. When a planet forms in a disk, angular momentum has to be carried away from the planet otherwise its rotation speed would be far too large. Only turbulence can achieve such a large angular momentum transport. Recent studies mentioned that, at fixed value of the Froude number, $F r=\Omega_{i} / N$, with $N=\sqrt{g \alpha \partial T / \partial z}$ the buoyancy frequency and $\Omega_{i}$ the angular velocity of the inner cylinder, the system is more stable when increasing the Reynolds number $R e=\Omega_{i} R_{i}\left(R_{0}-R_{i}\right) / v[30,31]$. The final objective is to eventually explore the not yet understood turbulent flows, to get closer to actual astrophysical applications, by taking advantage of optimized HPC.

Baroclinic instability is recognized to be one of the dominant energetic processes in the large-scale atmospheres of terrestrial planets, such as the Earth and Mars [32], and in the oceans [33]. Its time-dependent behaviour exerts a dominant influence on the intrinsic predictability of the atmospheric circulation and the degree of chaotic variability in its large-scale meteorology [32,34,35]. Inertia gravity waves (IGWs) are ubiquitous in the atmosphere and the oceans and are known to play a fundamental role in a wide variety of processes. The contributions mainly concern the transport of a significant amount of energy and momentum, the initiation and organization of convection, the induction and modulation of turbulence, as well as the modification of the mean circulation and thermal structure of atmospheric and oceanic motions [36]. Observations and simulations have revealed their spontaneous occurrence during the development of baroclinic instability. In spite of intensive research activities carried out over the last decades, the generation mechanism and the propagation of IGWs, as well as their interaction with large-scale structures, remain poorly understood. A better understanding of these phenomena is therefore mandatory for the improvement of the IGW parameterization schemes actually required to upgrade numerical global weather predictions. Since the pioneering works of Hide [12], the differentially heated, rotating cylindrical annulus has been an archetypal means of studying the properties of fully-developed baroclinic instability in the laboratory. Recent works mainly based on direct numerical simulations reported the occurrence of IGWs in water-filled baroclinic cavity along the inner cold cylinder [37-39]. Moreover, Von Larcher et al. [39] observed the presence of additional small-scale ripples resulting from hydrodynamical instability along the hot wall.

The paper is organized as follows. First, the physical and mathematical models of the SRI and Baroclinic configurations are detailed. The numerical methods to solve the coupled Navier-Stokes and energy equations in annuli are introduced before describing the parallelizations strategies. The last section is devoted to the validation of the new numerical solver. Accuracy will be checked, validations with existing findings for strato-rotational and baroclinic instabilities are performed including a strong scaling test.

\section{Physical and mathematical models}

\subsection{Physical model}

This study focuses on flows in rotating annular cavities filled with an incompressible fluid and submitted to either a radial or a vertical thermal gradient. In these configurations, the flow results from the competition between the rotation of the cylinders and the buoyancy force arising from the prescribed temperature gradient.

The two configurations considered together with the notations are depicted in Fig. 1.

The first configuration corresponds to a Taylor-Couette system (Fig. 1a) where the fluid is submitted to a stable vertical thermal gradient, which means that the top boundary is heated whereas the bottom one is cooled. The inner cylinder rotates faster than the outer one. To reduce the edge effects, stress-free conditions are prescribed for velocity on the two horizontal disks. Despite the stabilizing effect of the vertical stratification induced by the stable thermal gradient, an hydrodynamic instability, named the strato-rotational instability, may develop for specific values of the 


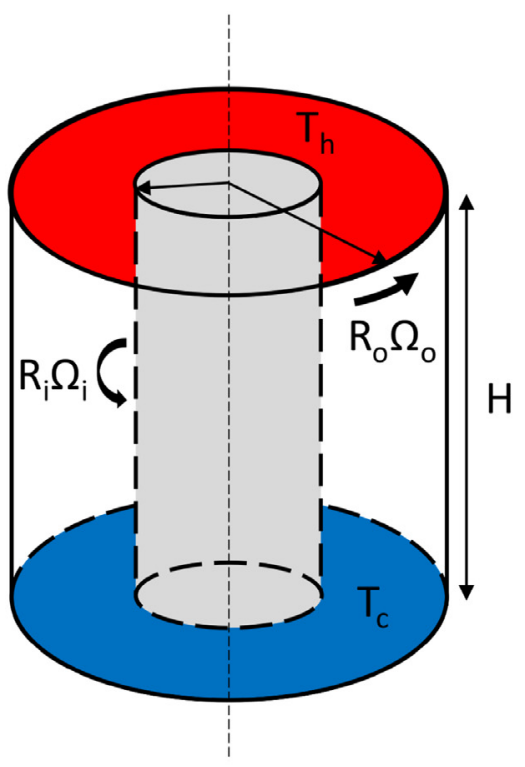

(a) SRI configuration

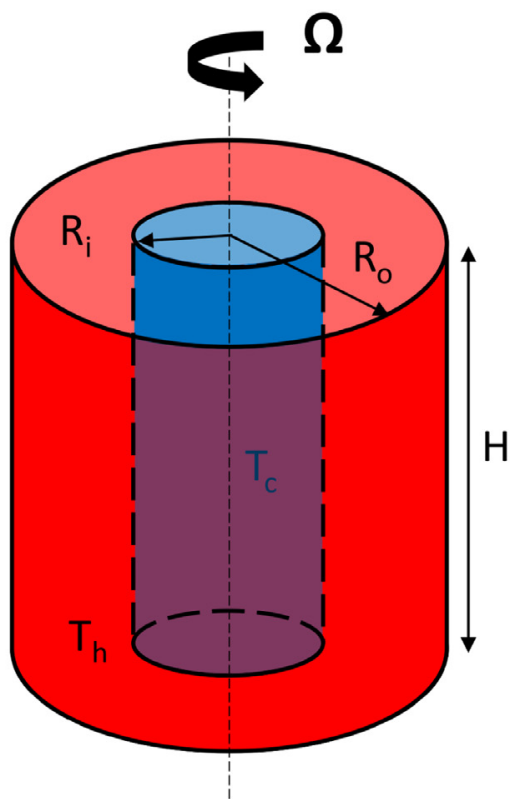

(b) Baroclinic configuration

Fig. 1. Rotating annular cavities configurations.

control parameters which are the angular speed ratio $\mu$, the Reynolds number Re and the Froude number Fr defined by:

$\mu=\frac{\Omega_{0}}{\Omega_{i}}, \quad \operatorname{Re}=\frac{\Omega_{i} d R_{i}}{v}, \quad F r=\frac{\Omega_{i}}{N}$

with $d=R_{0}-R_{i}, v$ the kinematic viscosity, $\Omega_{i}$ and $\Omega_{0}$ the angular speeds of the inner and outer cylinders respectively, and $N$ the Brunt-Väisälä frequency which is a measure of the vertical stratification scale. In the framework of the Boussinesq approximation (see section 2.2), $N$ is defined by:

$$
N^{2}=\frac{\alpha g \Delta T}{H}
$$

with $\alpha$ the thermal expansion coefficient.

In the second configuration (Fig. 1b), a radial temperature gradient is prescribed by heating the outer cylinder and cooling the inner one. Unlike the previous case, the two vertical cylinders rotate with the same angular velocity $\Omega$. The top boundary is an open free surface. This configuration was found to be a suitable testbed for studying mid-latitude atmospheric flows where a baroclinic instability together with inertia gravity waves may develop. The classical control parameters are the Taylor number $\mathrm{Ta}$ and the Rossby number Ro [12], defined by:

$T a=\frac{4 \Omega^{2} d^{5}}{v^{2} H}, \quad R o=\frac{\alpha \Delta T g H}{\Omega^{2} d^{2}}$

\subsection{Mathematical model}

In both configurations, the flow and heat transfer are governed by the incompressible Navier-Stokes equations coupled with energy equation through the Boussinesq approximation. With these assumptions, the set of equations reads:

$\begin{cases}\nabla \cdot \mathbf{u}=0 & \text { in D } \\ \partial_{t} \mathbf{u}+\frac{1}{2}[(\mathbf{u} . \nabla) \mathbf{u}+\nabla .(\mathbf{u u})]=-\nabla p+v \Delta \mathbf{u}+\mathbf{F} & \text { in D } \\ \partial_{t} T+\frac{1}{2}[(\mathbf{u} \cdot \nabla) T+\nabla \cdot(\mathbf{u} T)]=\kappa \nabla^{2} T & \text { in D }\end{cases}$

$D$ being the computational domain, $\kappa$ the fluid thermal diffusivity and $\mathbf{u}=\left(u_{r}, u_{\theta}, u_{z}\right), p$ and $T$ standing for the velocity, pressure and temperature fields, respectively. The vectorial Laplacian
Table 1

Source terms $\mathbf{F}$ and boundary conditions in the SRI and baroclinic flows ( $T_{h}$ and $T_{c}$ stand for the hot and cold temperatures)

\begin{tabular}{lll}
\hline & Vertical $\nabla T$ & Radial $\nabla T$ \\
\hline $\mathbf{F}$ & $\alpha\left(T-T_{0}\right) \mathbf{g}$ & $\alpha\left(T-T_{0}\right) \mathbf{g}+2 \boldsymbol{\Omega} \times \mathbf{u}+\mathbf{\Omega} \times(\boldsymbol{\Omega} \times \mathbf{r})$ \\
Top wall & Free stress & Free stress \\
Bottom wall & Free stress & No-slip \\
Lateral walls & Differential rotation & No-slip \\
Top wall & $T_{h}$ & Adiabatic \\
Bottom wall & $T_{c}$ & Adiabatic \\
Inner wall & Adiabatic & $T_{c}$ \\
Outer wall & Adiabatic & $T_{h}$ \\
\hline
\end{tabular}

$\Delta \mathbf{u}$ expressed in cylindrical coordinates is given by the following formula:

$\Delta \mathbf{u}=\left(\begin{array}{l}\nabla^{2}\left(u_{r}\right)-u_{r} / r^{2}-\left(2 / r^{2}\right) \partial_{\theta} u_{\theta} \\ \nabla^{2}\left(u_{\theta}\right)-u_{\theta} / r^{2}+\left(2 / r^{2}\right) \partial_{\theta} u_{r} \\ \nabla^{2}\left(u_{z}\right)\end{array}\right)$

where $\nabla^{2}($.$) stands for the scalar Laplacian defined by:$

$\partial_{r}^{2}+(1 / r) \partial_{r}+\left(1 / r^{2}\right) \partial_{\theta}^{2}+\partial_{z}^{2}$

In Eq. (4), the source term $\mathbf{F}$ accounts for external forces, mainly the buoyancy force. For the first case corresponding to a TaylorCouette configuration (Fig. 1a), the equations are expressed in a fixed frame. For the second configuration with a radial thermal gradient (Fig. 1b), the equations are solved in a rotating reference frame and, as a consequence, in addition to the buoyancy force, Coriolis and centrifugal forces are also included in $\mathbf{F}$. The expression of $\mathbf{F}$ and the boundary conditions are given in Table 1 for the two configurations.

\section{Numerical method}

\subsection{Time discretization and projection scheme}

The second order semi-implicit Adams-Bashforth/BackwardEuler scheme is used for the time advancement of Eq. (4). The 
diffusive terms are implicitly treated except for the crossed terms of the vectorial Laplacian, resulting from cylindrical coordinates, which are explicitly evaluated. The temporal scheme reads:

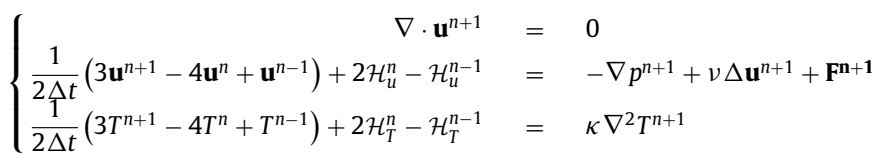

where $\mathcal{H}_{u}$ and $\mathcal{H}_{T}$ account for non-linear convective terms. For the momentum equations, the crossed contributions in the diffusive term are also included in $\mathcal{H}_{u}$. The velocity-pressure coupling is solved by a projection algorithm. Specifically, we used the improved method proposed by Hugues and Randriamampianina [27] which allows a temporal evolution of the normal pressure gradient at boundaries. This projection algorithm is detailed in $[27,40]$. Thus, the discretized Boussinesq-Navier-Stokes equations (7) reduce to two Poisson problems for a preliminary pressure and a pressure correction respectively, and four Helmholtz problems arising from the momentum and energy equations. The accuracy and the computational efficiency rely on the choice of the space discretization. In this work, we choose the spectral discretization in the azimuthal direction, using Fourier series, and the compact finite differences scheme in the two other directions. A short description is given hereafter.

\subsection{Compact scheme discretization}

The spatial approximation in the non-periodic $r$ and $z$ directions of the different terms of Eq. (4) is based on a fourth-order compact scheme discretization $[24,41,42]$. Compact schemes have a better resolution at high wavenumber than explicit finite differences. However, compact schemes induce a computational overhead due to the solution of tridiagonal/pentadiagonal linear systems. This overhead remains acceptable since the algorithm complexity is linear. A staggered grid arrangement of the velocity and the pressure is considered to ensure some conservation properties $[42,43]$. For instance, in the radial direction, the grid in the interval $\left(R_{i}, R_{0}\right)$ is composed of the pressure nodes, $r_{i+1 / 2}=R_{i}+(i-1 / 2) h_{r}$ with $0 \leq i \leq n_{r}+1$, and of the velocity nodes $r_{i}=i h_{r}$ with $0 \leq i \leq n_{r}$. The uniform space step is $h_{r}=\left(R_{o}-R_{i}\right) / n_{r}$. Non-uniform grids are obtained by means of an analytical mesh transformation, introducing the metric coefficients which are analytically computed [41]. The staggered fourth-order compact scheme approximations from the pressure to velocity nodes, reads for the staggered derivation:

$\alpha f_{i-1}^{\prime}+f_{i}^{\prime}+\alpha f_{i+1}^{\prime}=a\left(f_{i+1 / 2}-f_{i-1 / 2}\right) / h_{r}$

and for the staggered interpolation:

$\alpha f_{i-1}+f_{i}+\alpha f_{i+1}=a\left(f_{i+1 / 2}+f_{i-1 / 2}\right) / 2$

where the coefficients $\alpha$ and $a$ are calculated from accuracy requirements [24]. The boundary condition relations are:

$\left\{\begin{array}{c}f_{1}^{(p)}+\alpha f_{2}^{(p)}=a f_{-1 / 2}+b f_{1 / 2}+c f_{3 / 2} \\ f_{n}^{(p)}+\alpha f_{n-1}^{(p)}=a f_{n+1 / 2}+b f_{n-1 / 2}+c f_{n-3 / 2}\end{array}\right.$

The implicit nature of this finite difference scheme is expressed by the equivalent matrix formulation $M f^{(p)}=B f$ of Eqs. (9) and (10) where superscript $p$ refers to either derivation (') $p=1$ or interpolation $p=0$, and $M$ refers to a square tridiagonal matrix. All the coefficients defining the fourth-order accurate compact scheme operators used in this study are detailed in Table 2.

\subsection{The full diagonalization method}

This section presents the full diagonalization method used for solving the Helmholtz/Poisson problems (Eq. (7)) which is an efficient direct solver for linear systems arising from higher-order
Table 2

Compact scheme coefficients.

\begin{tabular}{llllllll}
\hline & \multicolumn{2}{l}{ Inner nodes } & & \multicolumn{3}{l}{ Boundary nodes } \\
\cline { 2 - 3 } \cline { 6 - 7 } Operators & $\alpha$ & $a$ & & $\alpha$ & $a$ & $b$ & $c$ \\
\hline $\mathrm{D}_{\xi}^{c f}$ & $1 / 22$ & $12 / 11$ & & -1 & -1 & 2 & -1 \\
$\mathrm{D}_{\xi}^{f c}$ & $1 / 22$ & $12 / 11$ & & 23 & -25 & 26 & -1 \\
$\mathrm{I}_{\xi}^{c f}$ & $1 / 6$ & $2 / 3$ & & 5 & $15 / 4$ & $5 / 2$ & $-1 / 4$ \\
$\mathrm{I}_{\xi}^{f c}$ & $1 / 6$ & $2 / 3$ & & 1 & $1 / 4$ & $3 / 2$ & $1 / 4$ \\
\hline
\end{tabular}

discretizations [26]. The main advantage of this approach is to eliminate the bad condition number of the operator matrices responsible for high computational costs with iterative solvers [44]. However, it is worth mentioning the three major drawbacks of the full diagonalization method which are the high algorithm complexity, the limitation to constant coefficients, and the required tensorial framework. For the sake of clarity of the following sections, this linear solver is detailed in the case only for a Poisson equation in cylindrical coordinates. With the tensorial notations and using Fourier series expansion, we have to compute for each Fourier mode $i$ the following 2D solution:

$\left(\mathrm{D}_{i r}^{2} \otimes \mathrm{I}_{z}+\mathrm{I}_{r} \otimes \mathrm{D}_{z}^{2}\right) \hat{\phi}_{i}=\hat{s}_{i}, \quad 0 \leq i \leq n_{\theta} / 2$

where the operators $\mathrm{D}_{\text {ir }}^{2}$ and $\mathrm{D}_{z}^{2}$ are defined by:

$\mathrm{D}_{i r}^{2}=\frac{1}{r} \delta_{r}^{f c} r \delta_{r}^{c f}-\frac{i^{2}}{r^{2}}, \quad \mathrm{D}_{z}^{2}=\delta_{z}^{f c} \delta_{z}^{c f}$

and $\hat{s}_{i}$ represents the $i$ th mode of the 1D Fourier transform in the azimuthal direction of the source term $s$ denoted by $\mathcal{F}_{\theta}(s)$. It should be noted that these operators are modified to include boundary conditions [41] and that the radial operator depends on the wavenumber $i$. The full diagonalization method consists of a two step method. Consequently, in the first step named preprocessing stage, the eigenvalues $(\Lambda)$ and eigenvectors $(P)$ of the operators are computed such as $\mathrm{D}_{i r}^{2}=\mathrm{P}_{i r}^{-1} \Lambda_{i r} \mathrm{P}_{i r}$ and $\mathrm{D}_{z}^{2}=\mathrm{P}_{z}^{-1} \Lambda_{z} \mathrm{P}_{z}$. This expensive step is performed only once during the preprocessing. During the processing stage the solution is computed at each time step. It involves fast Fourier transforms and tensorial/pointwise products for each wavenumber of the Fourier transform as follows:

1. compute the forward FFT of the source term: $\hat{S}=\mathcal{F}_{\theta}(S)$.

2. perform tensorial product $F_{i}=\left(\mathrm{P}_{i r}^{-1} \otimes \mathrm{P}_{z}^{-1}\right) \hat{S}_{i}$

3. compute pointwise product $F_{i j k}=F_{i j k} /\left(\lambda_{i r, j}+\lambda_{z, k}\right)$

4. perform tensorial product $F_{i}=\left(\mathrm{P}_{i r} \otimes \mathrm{P}_{z}\right) F_{i}$

5. compute the backward FFT to get the solution $\phi=\mathcal{F}_{\theta}^{-1} F$

The second step involves 2 Fast Fourier Transforms and 4 tensorial products. The algorithm complexity of this linear solver is $\mathcal{O}\left(\left(n_{r}^{2} n_{z}+n_{r} n_{z}^{2}+n_{r} n_{z} \log n_{\theta}\right) n_{\theta}\right)$. Thus, the Fourier series expansion in the azimuthal direction contributes to reduce computational resources, otherwise a full 3D solver has a higher linear complexity $\mathcal{O}\left(n_{\theta} n_{r}^{2} n_{z}+n_{\theta} n_{r} n_{z}^{2}+n_{r} n_{z} n_{\theta}^{2}\right)$.

\subsection{Code parallelization}

The parallelization of the present code relies on two distinct strategies: to evaluate the compact derivative/interpolation and to get solutions of Poisson and equation problem [10]. Both approaches are described in the following sections.

\subsubsection{Helmholtz/Poisson solvers}

First, the parallelization of the full diagonalization method still remains a tricky task. Indeed, the core of the method is mainly based on tensorial products to cross between the physical and 


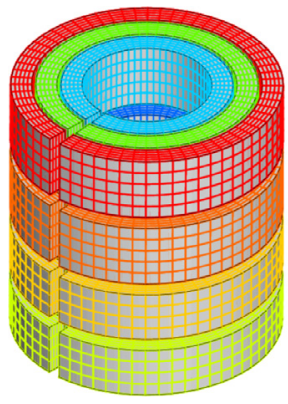

(a) $\theta$-pencil

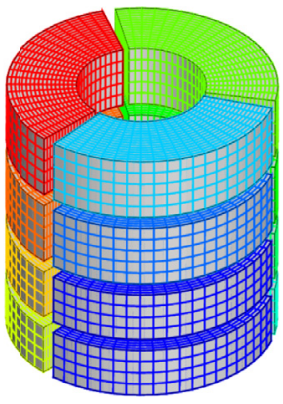

(b) $r$-pencil

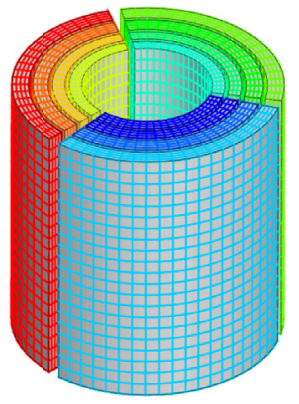

(c) $z$-pencil

Fig. 2. The 3 pencil decomposition states defined on $3 \times 4$ processor grids.

eigenvectors spaces, and the lack of sparsity in the eigenvector matrices involves expensive communications. Here, the 2d-pencil decomposition is considered to get a parallel version of the full diagonalization method [10]. The guideline of the 2d-pencil decomposition is to provide a 2D block decomposition where processors are filled into a 2D grid. Two of the dimensions of 3D data arrays are distributed on the processor grid. Such a decomposition leads to entirely maintain one dimension of the grid within the local memory. On this former direction, FFT or tensorial products can be performed in a serial way. An example of such an implementation is provided by the libraries P3DFFT or 2DECOMPFFT [45,46]. Fig. 2 illustrates the 2d-pencil decomposition in the cylindrical configuration, and the three pencil states.

Global data transposes are performed to switch between the three states inducing expensive MPI-all-to-all communications. The parallel algorithm of the diagonalization method can be summarized as follows:

1. compute $\hat{S}=\mathcal{F}_{\theta}(S)$ on the $\theta$-pencil state,

2. transpose $\hat{S}$ from $\theta$ to $r$-pencil then compute $F_{i}=\left(P_{i r}^{-1} \otimes \mathrm{I}_{z}\right) \hat{S}_{i}$

3. transpose $\hat{F}$ from $r$ to $z$-pencil then compute $G_{i}=\left(\mathrm{I}_{r} \otimes P_{z}^{-1}\right) F_{i}$

4. compute $F_{i j k}=G_{i j k} /\left(\lambda_{i r, j}+\lambda_{z, k}\right)$

5. compute $G_{i}=\left(\mathrm{I}_{r} \otimes P_{z}\right) F_{i}$ then transpose $G$ from $z$ to $r$-pencil,

6. compute $F_{i}=\left(P_{i r} \otimes \mathrm{I}_{z}\right) G_{i}$ then transpose $F$ from $r$ to $\theta$-pencil,

7. compute the final solution using a backward FTT: $\phi=$ $\mathcal{F}_{\theta}^{-1}(F)$

The previous algorithm involves 4 all-to-all communications during steps (2-3) while (5-6) are provided by a $2 \mathrm{~d}$-pencil decomposition library. Due to the implicit nature of the boundary conditions [41] two additional transpositions are required. Therefore, the total number of all-to-all communications necessary to solve each Helmholtz/Poisson problem is 6. Although the all-to-all communications are expensive, this approach exhibits a correct strong scaling [10] and contributes to drastically reduce the real execution time of a simulation. However, it should be stressed that a poor weak scaling is expected due to the $\mathcal{O}\left(n^{4}\right)$ algorithmic complexity of the diagonalization method. This is inconsistent with the concept of weak scaling, unlike methods with $\mathcal{O}(n)$ algorithmic complexity as the multigrid methods.

\subsubsection{Derivative and interpolation computations}

The spectral evaluation of derivatives and interpolations in the periodic direction $\theta$ involves real-to-complex and complex-to-real FFT. Computations are made in a serial manner since the default pencil-state is the $\theta$-pencil (Fig. 2). The reduced Partial Diagonal Dominant (rPDD) algorithm of Sun [25] underlies the computation of the compact derivatives and interpolations in the present approach. This method of solving tridiagonal linear systems shows interesting parallel performances $[10,25]$. The diagonal dominance is exploited to derive an approximate solver involving only neighborto-neighbor communications, greatly improving the parallel efficiency. Specifically, $2 \times 2$ linear systems involving interface nodes (reduced system) is derived by dropping terms through the diagonal dominance property [25]. The analysis and the reliability of the rPPD algorithm in the specific framework of the incompressible Navier-Stokes equations in cartesian coordinates is detailed by [10]: a description is briefly recalled hereafter. The calculation of a compact scheme derivative like Eqs. (9) and (10) using rPDD , consists of a three-step algorithm involving mainly the solution of the distributed tridiagonal linear systems $M_{p}$ on $P$ processors. These steps are summarized hereafter:

1. compute an approximate compact scheme derivative $\tilde{u}_{p}^{\prime}=$ $M_{p}^{-1} B_{p} u_{p}, p$ standing for a subdomain partition,

2. exchange boundary informations (neighbor-to-neighbor communications) and compute solutions of the reduced systems,

3. update the solution $\tilde{u}_{p}^{\prime}$ from solutions of the reduced systems independently on each processor.

The accuracy of this approximation depends on the diagonal dominance of the linear system [25]. For the fourth-order compact schemes (Table 2), it is shown that using about 16 nodes per subdomain does not bring significant deterioration of either the accuracy or conservation properties. A good strong scaling has been observed for up to $10^{4}$ processors [10]. It is worth mentioning that the staggered grid layout involves about 20 nodes for the convective terms which would require naively about 80 all-to-all mpicommunications with the pencil-decomposition strategy. In conclusion, the main advantage of the reduced PDD algorithm is to address a less intensive parallel communication than the pencil decomposition.

\section{Results and discussions}

This section investigates the effects of the rPDD algorithm within the context of rotating flows, and estimates its parallel performances. First, the accuracy of the temporal and spatial schemes is demonstrated over an analytical solution of the Navier-Stokes equations. Then, results for the two configurations described in Section 2.1 are presented to assess the rPDD algorithm performance and its parallel efficiency.

\subsection{Numerical accuracy}

Both spatial and temporal accuracies are determined with the Method of Manufactured Solutions (MMS) [47]. A fluid of viscosity $v=0.1$ is considered in an annular cavity defined by $\eta=R_{i} / R_{0}=$ $0.5, d=R_{0}-R_{i}=1$ and $\Gamma=H / d=2$. According to the MMS, a source term is derived from an analytical divergence-free velocity 


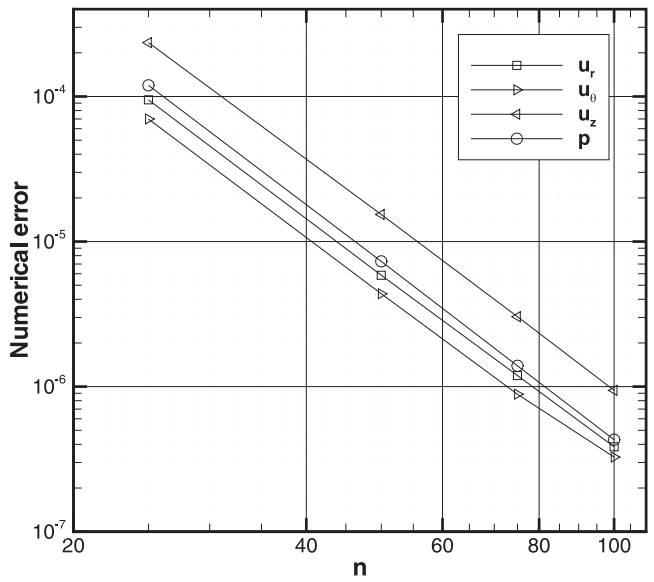

(a)

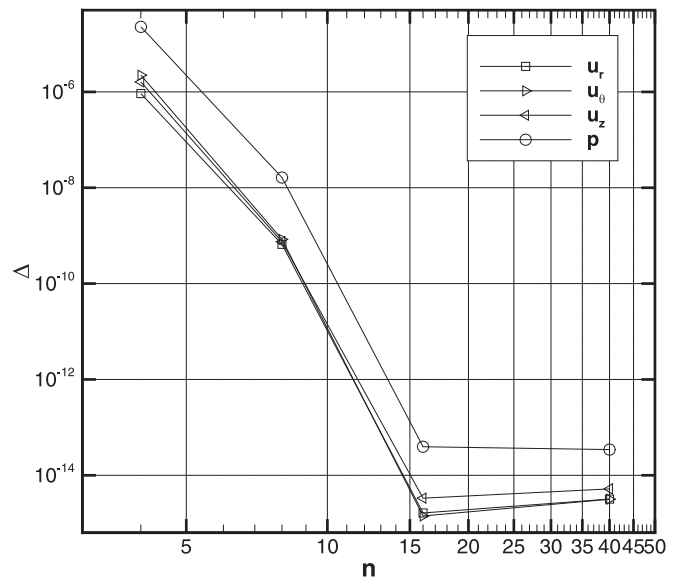

(b)

Fig. 3. Accuracy assessment of the space discretization scheme (a) the numerical errors versus size mesh, (b) difference error versus the partition size.

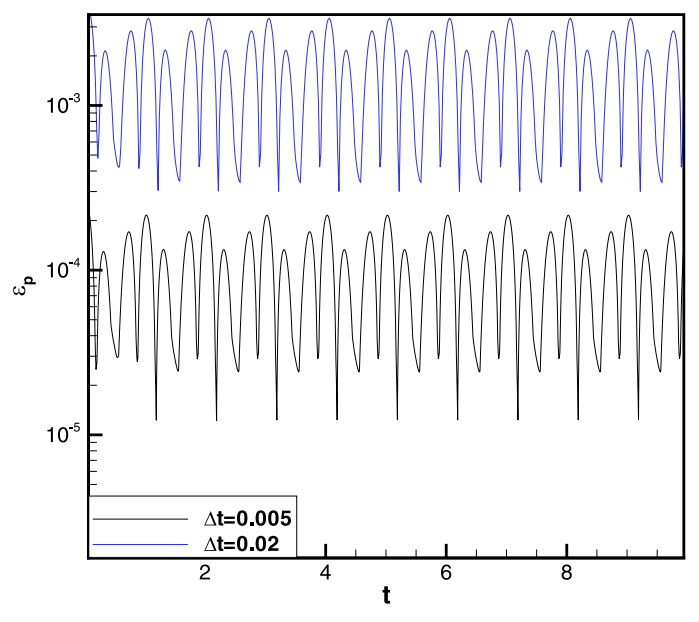

(a)

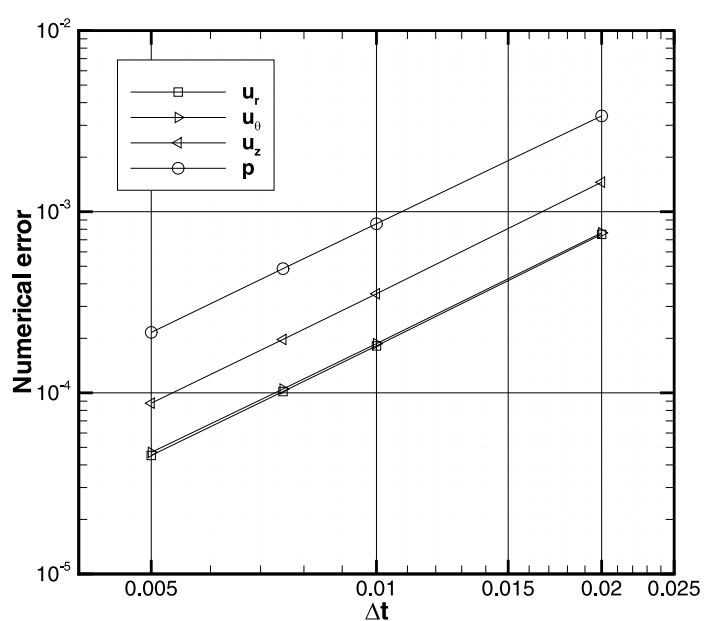

(b)

Fig. 4. Accuracy assessment of the temporal scheme (a) time history of the numerical errors, (b) maximum of the numerical error versus time step.

and associated pressure fields satisfying the Navier-Stokes equations. Here, the following divergence-free velocity and pressure fields have been considered:

$u_{r}=+\frac{1}{2 \pi} \sin ^{2}\left(\pi\left(r-r_{i}\right) / d\right) \cos (\theta) \sin (2 \pi z / H)$

$(1+\beta \cos (2 \pi t))$

$u_{\theta}=-\frac{1}{2 \pi} \sin ^{2}\left(\pi\left(r-r_{i}\right) / d\right) \sin (\theta) \sin (2 \pi z / H)$

$$
(1+\beta \cos (2 \pi t))
$$

$$
\begin{aligned}
u_{z}= & -\frac{H}{2 \pi d} \sin ^{2}\left(2 \pi\left(r-r_{i}\right) / d\right) \cos (\theta) \sin (\pi z / H) \\
& (1+\beta \cos (2 \pi t))
\end{aligned}
$$

$$
\begin{aligned}
p= & \left(\sin \left(\pi\left(r-r_{i}\right) / d\right)+\sin (\pi z / H)\right) \cos (\theta) \\
& (1+\beta \cos (2 \pi t))
\end{aligned}
$$

The steady solution is obtained for $\beta=0$, whereas $\beta=1$ leads to unsteady solution. The discussion on the numerical accuracy is based on the maximum relative error:

$\epsilon_{\phi}(t)=\frac{\left\|\phi_{\text {num }}-\phi_{\text {ex }}\right\|_{\infty}}{\left\|\phi_{\text {ex }}\right\|_{\infty}}$

where $\phi_{\text {num }}$ and $\phi_{\text {ex }}$ stand respectively for the numerical and analytical fields of the velocity components and the pressure.

First, the space accuracy is estimated from error (17) using various grids with increasing sizes. Tests are performed with the steady solution $(\beta=0)$, and the numerical error for each variable is reported in Fig. 3a.

The numerical error decreases with a -4 slope corresponding to the expected accuracy of the present compact scheme discretization.

The temporal accuracy is then evaluated by considering the unsteady solution $(\beta=1)$ leading to a time-dependent maximum relative error. The time-accuracy analysis is performed on the maximum value of this error during several periods. Fig. 4 shows the history of the numerical error and its maximum values after several periods versus the time step. Fig. 4 exhibits a -2 slope for each variable, in agreement with the expected order 2 of accuracy. These findings confirm that the accuracy requirements are well fulfilled by the present code. 


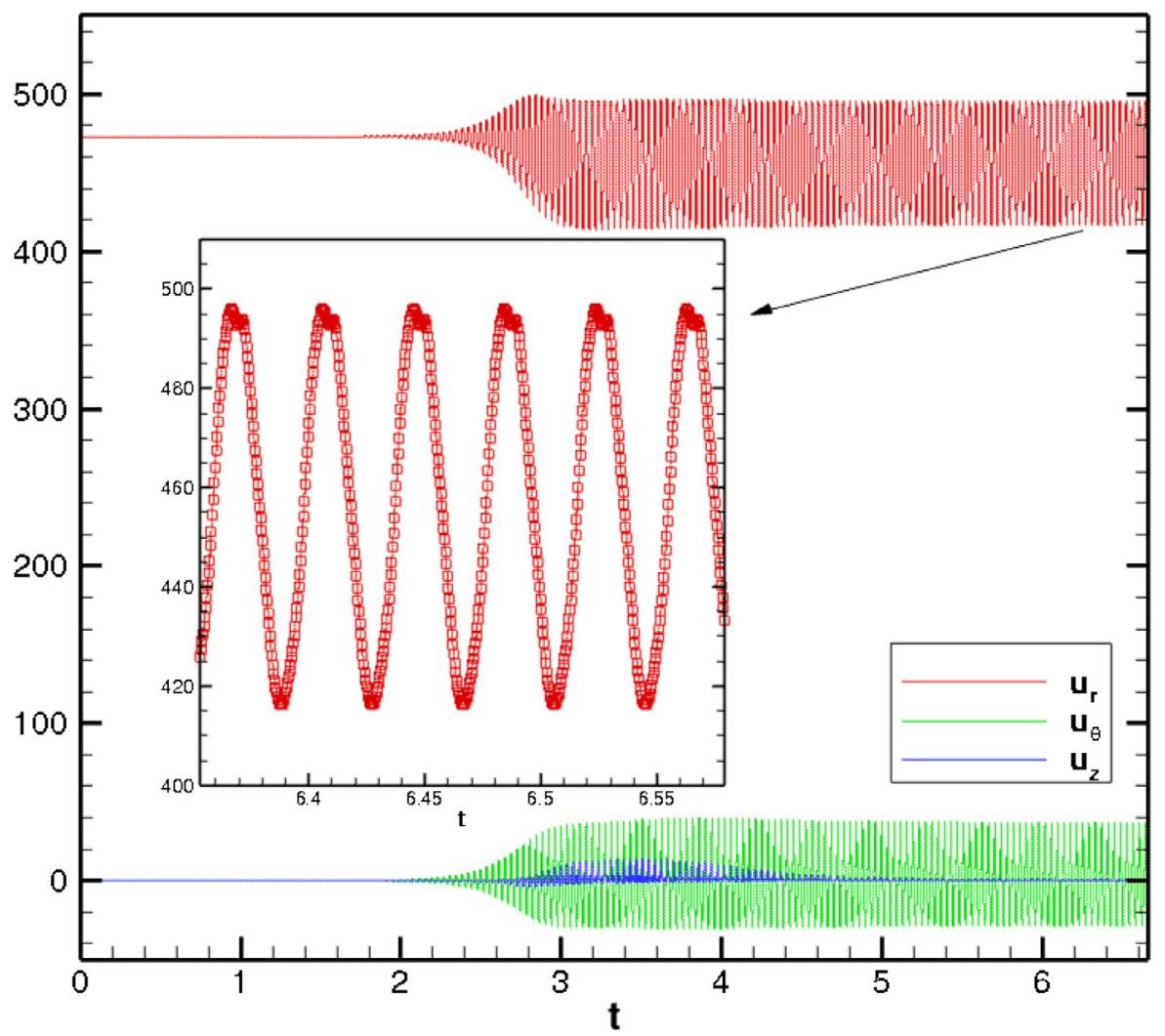

Fig. 5. Occurrence of the SRI: time history of the sampled velocity at mid-cavity for $(\operatorname{Re}, \operatorname{Pr}, F r, \mu)=(600,1,1.4,0.354)($ skipped symbol 20$)$.

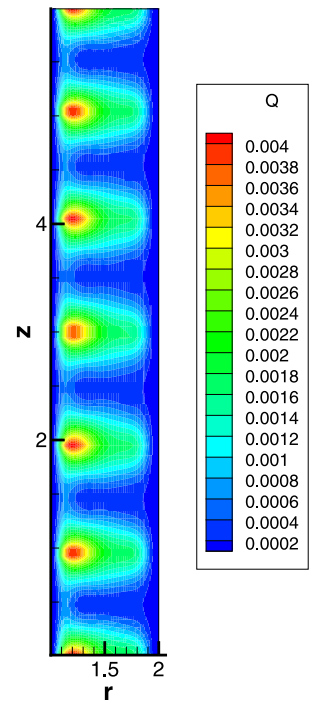

(a)

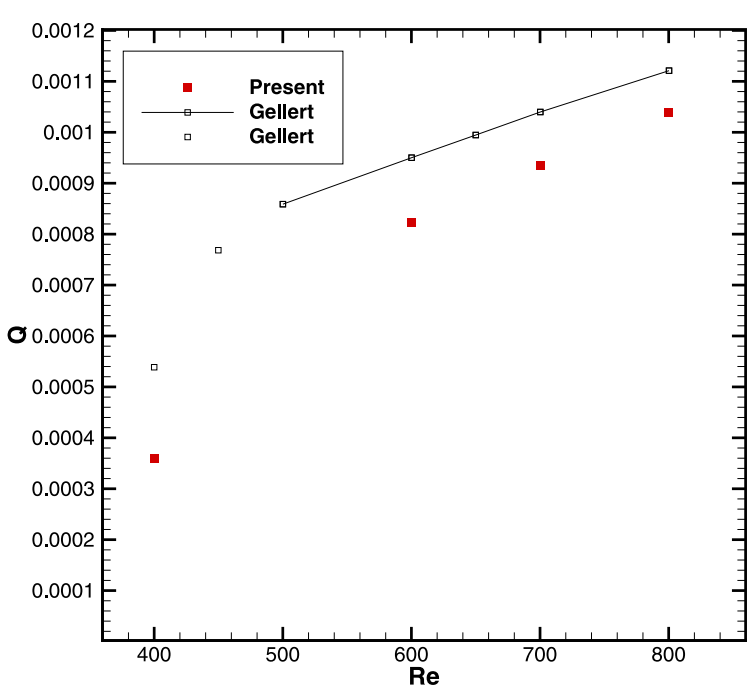

(b)

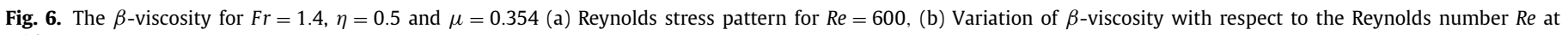
mid-gap.

The influence of the rPDD algorithm over the computed solutions is now investigated. For this purpose, node-wise comparisons between simulations using the rPDD and the classical ones are performed. It is recalled in Section 3 that the accuracy of the rPDD algorithm depends on the number of nodes involved in a partition. This parameter is denoted by $n / p$. Thus, the deviation introduced by the rPDD on the numerical solution is measured as:

$\Delta_{\phi}(n / p)=\frac{\left\|\phi_{r p d d}-\phi_{s t d}\right\|_{\infty}}{\left\|\phi_{s t d}\right\|_{\infty}}$ where std refers to the standard evaluation of compact scheme, or without error. In the following tests, the size number of the partition is kept identical in each non-periodic directions $(n / p=$ $\left.n_{r} / p_{r}=n_{z} / p_{z}\right)$. The maximum difference $\Delta_{\phi}$ versus the partition size $n / p$ is plotted in Fig. $3 \mathrm{~b}$. It is observed that the maximum difference drastically decreases with respect to the parameter $n / p$, in accordance with previous analysis $[10,25]$. This behaviour indicates that above a partition size $n / p=15$ no significant difference is observed, and consequently that the accuracy is not deteriorated. 
Table 3

Effects of the approximate rPDD algorithm at $R e=600$ for different node densities $n / p(n / p=96$ corresponds to the simulation without using PDD algorithm and $\mathrm{x}(\mathrm{m})$ denotes $x \times 10^{m}$ )

\begin{tabular}{lllllll}
\hline$n / p$ & $\Delta u_{r}$ & $\Delta u_{\phi}$ & $\Delta u_{z}$ & $\Delta T$ & $\nabla \cdot \mathbf{u}$ & $\Delta \beta$ \\
\hline 96 & - & - & - & - & - & $9.72(-12)$ \\
48 & $3.60(-11)$ & $3.20(-12)$ & $2.52(-11)$ & $3.14(-13)$ & $5.51(-13)$ & $1.19(-11)$ \\
24 & $3.51(-11)$ & $2.95(-12)$ & $2.63(-11)$ & $3.20(-13)$ & $5.20(-13)$ & $1.00(-11)$ \\
12 & $3.13(-09)$ & $3.34(-10)$ & $1.82(-09)$ & $8.09(-11)$ & $1.72(-10)$ & $1.00(-11)$ \\
6 & $2.98(-04)$ & $1.23(-04)$ & $2.67(-04)$ & $1.75(-05)$ & $3.22(-05)$ & $3.66(-06)$ \\
\hline
\end{tabular}

\subsection{Taylor-Couette flow with an axial temperature gradient}

Increased attention has recently been paid to the TaylorCouette flow with a vertical stably stratified temperature field. Indeed, despite the stabilizing differential rotation and thermal stratification, the flow may become unstable at specific values of the Reynolds and Froude numbers. This instability, named Stratorotational Instability (SRI), is suspected to be responsible for the outward angular momentum flux which is a necessary condition for materials to be transported inwards towards the central body in accretion disks. The major difficulty encountered in the numerical study of this configuration stems from the very long transient regime before the occurrence of the instability. As a consequence, simulation times to reach converged solutions are extremely lengthy, leading to huge computational times when using a sequential code. Moreover, the large aspect ratio in the axial direction, typical in this specific configuration, requires consequent number of modes, further increasing the simulation times. Thus, the present algorithm is a promising strategy to strongly reduce wall-clock times, without nevertheless reducing the algorithm complexity of the diagonalization method but excluding ideal weak scaling. For instance, the Fig. 5 illustrates the occurrence of a Strato-rotational instability by probing the velocity components at mid- height and radius of the cavity.

The converged solution requires about 300000 temporal iterations. In this section, several simulations are carried out to check the effects of the rPDD algorithm on the variation of the angular momentum transport.

We consider the flow in a tall annular cavity with $\eta=R_{i} / R_{0}=$ $0.5, d=R_{0}-R_{i}=1$ and $\Gamma=H /\left(R_{0}-R_{i}\right)=6$. The flow and heat transfer are governed by the dimensionless parameters $R e, F r$ and $\mu$ (see Section 2.1). The angular momentum is related to the normalized Reynolds stress, represented by the $\beta$ viscosity [11]:

$\beta=\frac{\left\langle u_{r}^{\prime} u_{\theta}^{\prime}\right\rangle}{R_{i}^{2} \Omega_{i}^{2}}$

where fluctuating velocities $u_{r}^{\prime}$ and $u_{\theta}^{\prime}$ correspond to deviations from the mean value in the azimuthal direction.

First, the $\beta$-viscosity is computed for $\operatorname{Re}=400,600,700$ and 800 with fixed values of parameters $(\mathrm{Pr}, \mathrm{Fr}, \mu)=(1,1.4,0.354)$. The mean $\beta$-viscosity along the $z$-axis is considered for comparison with the simulations of Gellert and Rüdiger [11] at mid-gap $r=1.5 d$. Computations were carried out on a grid size $48 \times 96 \times 96$ in $(r, \theta, z)$, found to be sufficient for describing the angular momentum. Fig. 6a shows the Reynolds stress pattern in a $r-z$ plane for $R e=600$. The evolution of the $\beta$-viscosity versus the Reynolds number is displayed in Fig. 6b. Despite a discrepancy from the profiles reported by Gellert and Rüdiger [11], which can be explained by the very different numerical techniques used including grid resolution, the linear dependence on the Reynolds number is recovered for $R e \geq 600$.

To investigate the effects of the approximation introduced by the rPDD algorithm (section 3.4.2), the $\beta$-viscosity is computed for several node densities $n / p$ at Reynolds number $R e=600$. Table 3 gathers the differences between the solutions obtained with and without the rPDD algorithm.

The rPDD algorithm is used for different partition size ranging from 48 nodes to 6 nodes per subdomain. The simulation performed with $n / p=96$ corresponds to the standard evaluation of the compact scheme derivatives. Table 3 reveals noticeable discrepancies on the velocity field and divergence below $n / p=12$ nodes per subdomain. A significant difference is obtained for 6 nodes per subdomain, where a difference of about $10^{-4}$ is observed. It should be mentioned that the divergence of the flow is around $510^{-12}$, and according to our experience this value corresponds to the threshold of the round-off error of the present simulation.

\subsection{Uniformly rotating flow with a radial temperature gradient: The baroclinic cavity}

The aim of this section is to assess the parallel performance of the present solver in the baroclinic cavity, in particular its ability to capture the different scales reported by Von Larcher et al. [39] within the same configuration. For this purpose, the flow in a differentially heated rotating annulus is considered, and more specifically the regime involving baroclinic instability. This flow configuration was extensively studied in the literature. Among others we can refer to the experimental work of Harlander et al. [48], the numerical simulations of von Larcher and Dörnbrack [49] and the recent benchmark of Vincze et al. [50]. The test case to validate the code is for a $6 \mathrm{rpm}$ rotation rate and with a radial temperature difference of $\Delta T=8 \mathrm{~K}$. The governing dimensionless parameters are the Taylor and the Rossby numbers defined by Eq. (3). In the following validation tests $T a=1.69 \times 10^{6}$ and $R o=0.2$.

Fig. 7a displays a snapshot of the free surface temperature showing the occurrence of the dominant azimuthal mode $m=3$ on the baroclinic instability. This wave- 3 structure is confirmed by the corresponding Hövmoller azimuth-time diagram at mid- radius and height, presented in Fig. 7b.

It is worth mentioning that the initial state to start the present simulation is an under-resolved solution $(64 \times 64 \times 64$ in radial, azimuthal and axial directions respectively) which was interpolated $(t=0)$ to a finer grid $256 \times 128 \times 150$. Both plots of Fig. 7 qualitatively show good agreement with previous results [50].

The Fig. 8 shows instantaneous isocontours in $(\theta, z)$ plane of the horizontal divergence of the velocity $\nabla_{h} \cdot \mathbf{u} \equiv-\frac{\partial w}{\partial z}$ along the inner cold wall (Fig. 8a) and along the outer hot cylinder (Fig. 8b). This variable is introduced to exhibit the occurrence of small-scale features simultaneously with the large-scale baroclinic waves. In the first plot, the small-scale structures, developing towards the bottom wall, have been identified as inertia gravity waves (IGWs) by different authors [37-39] in similar water-filled cavities. The present observed features recall such IGWs reported by these authors. Recently, for the same present configuration, Von Larcher et al. [39] mentioned the presence of ripples resulting from hydrodynamical instability along the outer hot cylinder. We also capture the same phenomenon, as illustrated by the isocontours of the horizontal divergence.

A quantitative validation is obtained from the comparison of the values of drift rate of the large-scale baroclinic waves. The drift rate $c_{m}(t)$ of a wave mode $m$ is calculated from the phase shift $\phi_{m}(t)$ at each time $t$ by :

$c_{m}(t)=\frac{1}{m} \frac{\mathrm{d} \phi_{m}}{\mathrm{~d} t}$

Fig. 9 shows the time history of the phase shift divided by the azimuthal wavenumber $m$.

The uniform drift for the dominant mode $m=3$ and the associated slave mode $m=6$ can thus be obtained. The predicted drift associated with the dominant mode $m=3$ is $c_{3}(t)=$ 


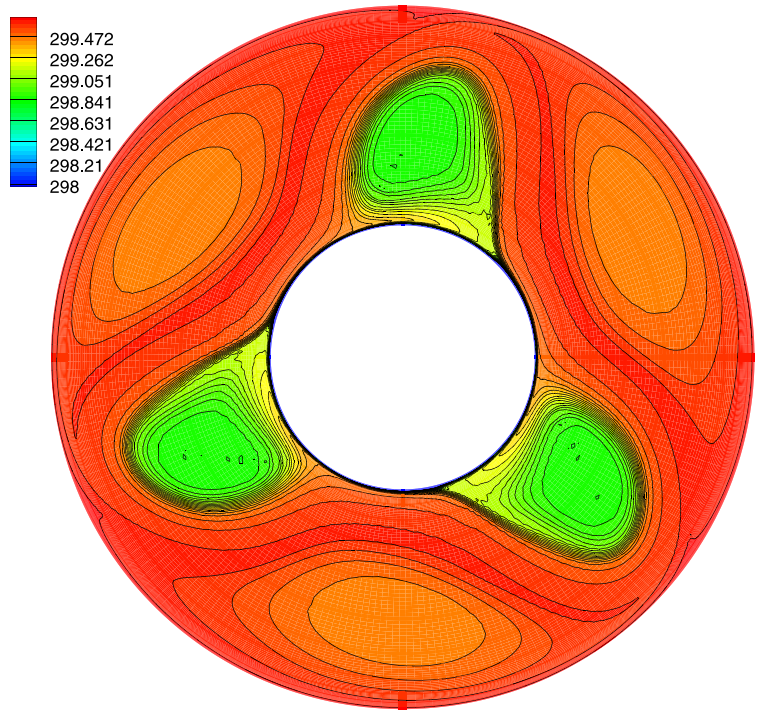

(a)

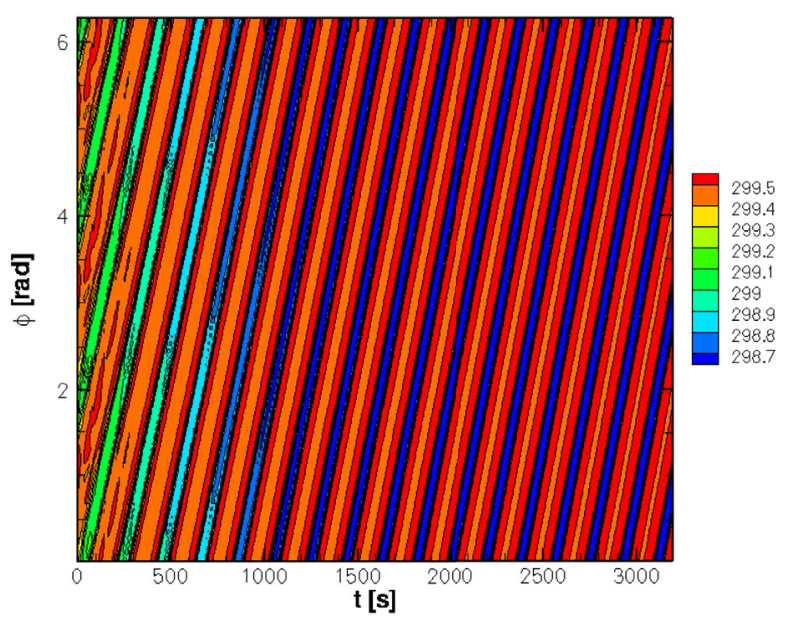

(b)

Fig. 7. Temperature at upper free-surface: (a) Snapshot (b) Hövmoller diagram at mid- radius and height in azimuth-time.

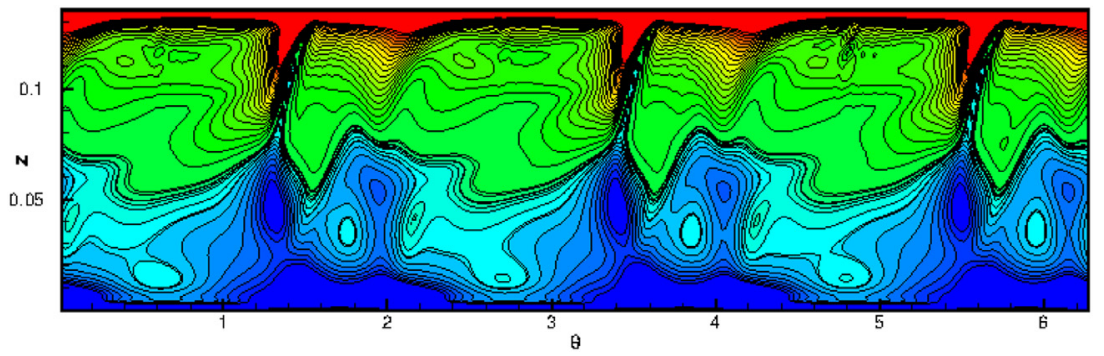

(a)

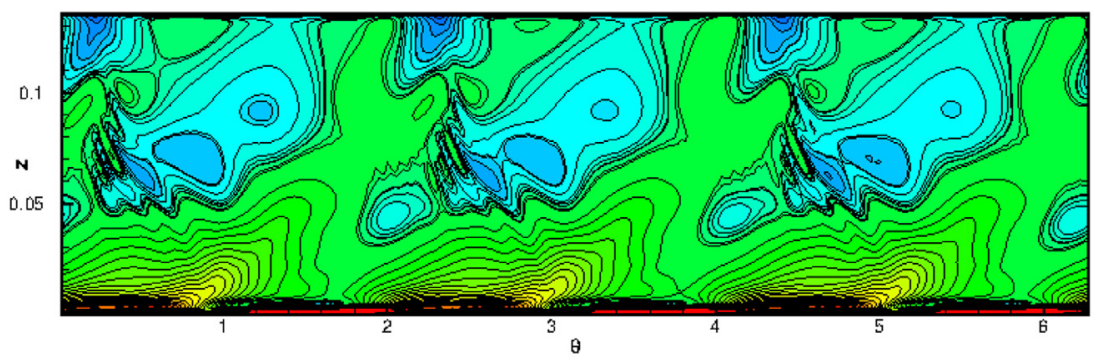

(b)

Fig. 8. Instantaneous isocontours in $(\theta, z)$ plane of the horizontal divergence of the velocity: at radius close to inner wall (a), at radius close to outer wall (b).

$0.0115 \mathrm{rad} . \mathrm{s}^{-1}$, which favorably compares with the values previously obtained experimentally and numerically (see Fig. (10) in [50]). The code being validated on the prediction of the baroclinic instability and associated small-scale features, we now focus on the parallel performance of the proposed method.

A strong scaling test is performed to assess the computational performance. It consists in measuring the computational time versus the number of cores. Three meshes are considered: $128 \times 128 \times 128,128 \times 264 \times 264$ and $128 \times 568 \times 568$. The mesh in the $r$ and $z$-directions is distributed on a square process grid of size $P^{2}$ ranging from 4 to 1024 cores. The computational costs to calculate the convective terms, $t_{c o n v}$, to solve a Poisson equation, $t_{\text {diag }}$, and to simulate one time step, $t_{i t}$, are measured. These values are presented in order to compare the performances of the two parallel strategies: the rPDD (explicit terms) and the 2d-pencil decomposition (implicit terms). The tests were performed on the
CALMIPs EOS cluster facilities which consists of 612 computation nodes of 2 Intel(r) IVYBRIDGE 2,8 GHz 10-cores. Fig. 10 shows the computational times versus the number of used cores.

For each mesh size, a similar behaviour is observed. The computational cost decreases with respect to the core number (Fig. 10a). For small core numbers, the scalability is satisfactory, but for the largest ones, a loss of scalability is observed with the coarser meshes. It is reasonable to speculate that this loss of performance will also occur with finer mesh for larger core numbers. This behaviour can be attributed to the ratio between the volume of computations per core and the data exchange involved in the MPI communications. Fig. 10b concerns the strong scaling test for the solution of a Poisson equation based on the $2 d$-pencil decomposition. The trend observed in Fig. 10a still holds. This can be explained by the large communication volume in the switch between two states of the 2d-pencil decomposition [18]. Indeed, a 


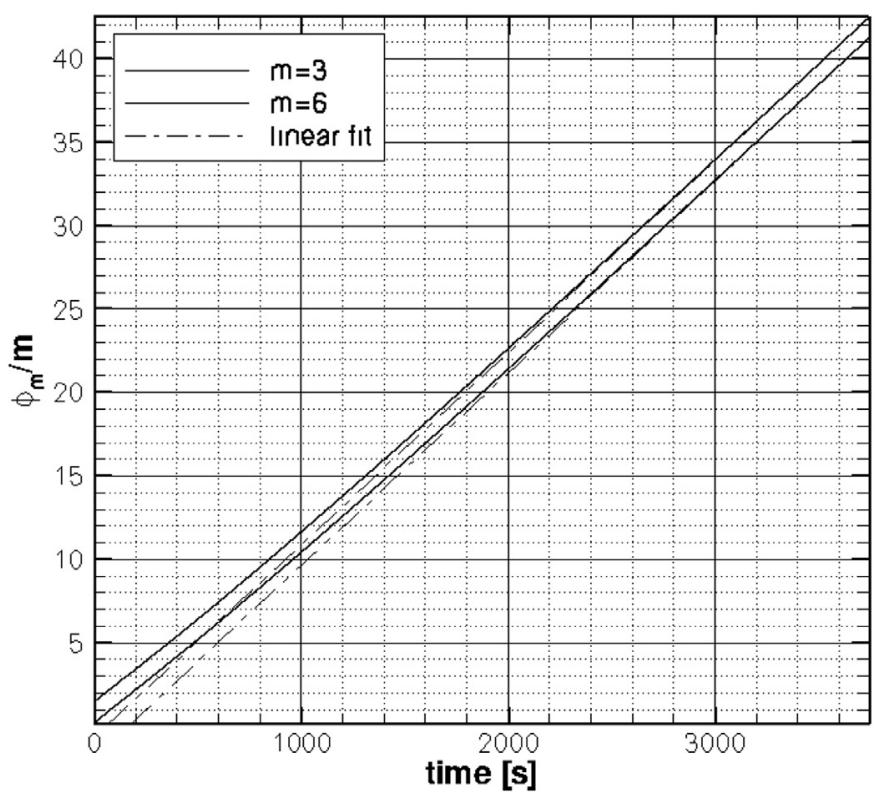

Fig. 9. Time history of the azimuthal distances $\Phi_{m} / m$ of wave modes $m=3$ and $m=6$.

global communication has to be tuned according to the cluster to get better efficiency. Fig. 10c shows the strong scaling test during the computation of convective terms. This computation relies only on the calculation of the compact interpolations and derivatives using the rPDD approach. In this case, a good scaling is noted for the two finest grids up to $10^{3}$ cores. In comparison with the $2 d-$ pencil decomposition, the rPDD involves only neighborhood communications which are less expensive. However, with the coarsest grid, the scalability from $10^{2}$ cores is deteriorated, which can be also explained by the unbalance between volume of computations and communications. Finally, it must be noted that the scalability and the computational time are dominated by the solution of Poisson/Helmholtz equations as shown by the similarities of the curves in Fig. (10a) and (10b). It should be noted that better performances are observed in Fig. 10b with the largest grid, wich may be explained by a memory cache effect. Even if a specific tuning can further improve performances, the present method exhibits a wall time reduction from $25 \mathrm{~s}$ with 4 cores to $0.35 \mathrm{~s}$ with 1024 cores when using a standard mesh of $128 \times 264 \times 264$.

\section{Conclusion}

In this manuscript, we have extended a cartesian parallel higher-order compact scheme solver to cylindrical coordinates. The azimuthal direction has been discretized using Fourier series expansion to benefit from the natural periodicity, and the favorable complexity algorithm of the Fast Fourier Transform. The space discretization in the two wall-bounded directions relies on the fourthorder compact scheme approximations. The guideline concerns the simulation of two specific configurations of rotating stratified flows: the Taylor-Couette setup under an axial thermal stratification and the baroclinic cavity. The code parallelization strategy combines two approaches. The first one is the $2 d$-pencil decomposition to address the parallel solution of the implicit viscous terms and the pressure-like equations based on the diagonalization method. The second strategy of parallelization consists in the calculation of the compact derivatives/interpolations. The approximate tridiagonal solver named reduced Partial Diagonal Dominant (rPDD) algorithm is used to evaluate compact derivatives and interpolations. According to our knowledge, the proposed parallelization strategy is a totally new method.

The present code is successfully validated against the Method of Manufactured Solution, and in the two test configurations. The effects of the approximation introduced by the rPDD on the solution accuracy is evaluated. Precisely, we show that with partition domains beyond 16 nodes the rPDD does not introduce noticeable error. Finally, a strong scaling test is performed to outline the potentiality of the present parallel strategy to simulate "specific rotating stratified flows". In particular, the present tool has allowed to drastically reduce the execution time for the two configurations, in comparison with sequential approach. Moreover, the proposed technique is able to correctly capture all the characteristic solution features : the SRI, the baroclinic instability and the associated small-scale structures (IGWs and outer ripples). Further exploration of these complex phenomena is now considered. It particularly concerns DNS of the turbulence in SRI. For the baroclinic configuration, the next studies should be dedicated to the large-tank facilities available at BTU, Cottbus-Senftenberg Germany (team of Professor U. Harlander). This setup allows for investigating IGWs in "atmosphere-like" situation with rotation rate smaller than thermal stratification. Nevertheless, the scalability test puts forward the pitfall introduced by the large communication scheme in the 2d-pencil decomposition. Performances could benefit from the hybrid OpenMP/MPI parallelization to enhance the computation/communication balance.

\section{Acknowledgements}

This work was granted access to the HPC resources of CALMIP supercomputing center under the allocation 2017P17027 and to the HPC resources of Aix-Marseille Université financed by the project Equip@Meso (ANR-10-EQPX-29-01) of the program "Investissements d'Avenir" supervised by the "Agence Nationale de la

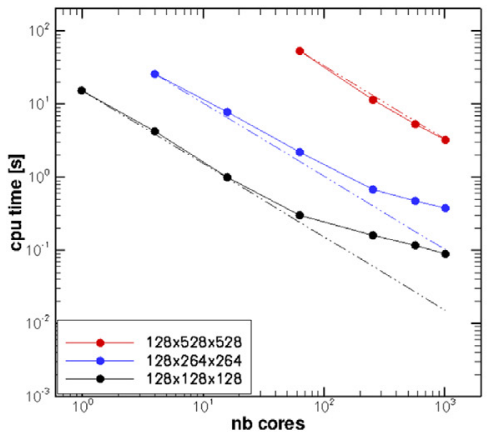

(a)

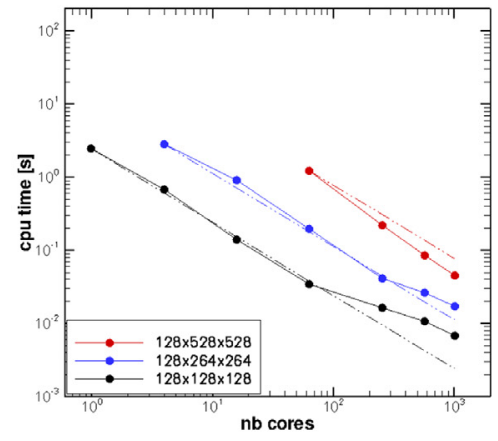

(b)

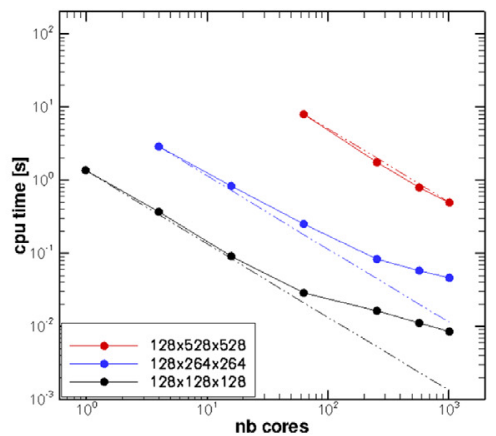

(c)

Fig. 10. Strong scaling test (a) temporal iteration (b) solution of Poisson equation (c) convective terms. 
Recherche". The authors acknowledge financial support from "Ministère des Affaires Etrangères", France, in the framework of PROCOPE projects (PHC 28369XH and PHC 35299PM). The authors are grateful to Pr U. Harlander (BTU, Cottbus-Senftenberg, Germany) for fruitful discussions.

\section{References}

[1] Yakovlev S, Moxey D, Kirby RM, Sherwin SJ. To CG or to HDG: a comparative study in 3d. J Sci Comput 2016;67(1):192-220.

[2] Witherden FD, Farrington AM, Vincent PE. PYFR: an open source framework for solving advection-diffusion type problems on streaming architectures using the flux reconstruction approach. Comput Phys Commun 2014; 185(11):3028-40.

[3] King J, Yakovlev S, Fu Z, Kirby RM, Sherwin SJ. Exploiting batch processing on streaming architectures to solve $2 d$ elliptic finite element problems: a hybridized discontinuous galerkin (HDG) case study. J Sci Comput 2014;60(2):457-82

[4] Moxey D, Cantwell C, Kirby R, Sherwin S. Optimising the performance of the spectral/hp element method with collective linear algebra operations. Comput Methods Appl Mech Eng 2016;310:628-45.

[5] Karniadakis G, Sherwin S. Spectral/hp element methods for computational fluid dynamics. Oxford University Press; 2013.

[6] Cantwell CD, Moxey D, Comerford A, Bolis A, Rocco G, Mengaldo G, et al. Nektar++: an open-source spectral/hp element framework. Comput Phys Commun 2015:192:205-19.

[7] Fischer P, Kruse J, Mullen J, Tufo H, Lottes J, Kerkemeier S. Nek5000: Open Source Spectral Element CFD Solver. Argonne National Laboratory, Mathematics and Computer Science Division, Argonne, IL; 2008. see https://nek5000 mcs.anl.gov/index.php/MainPage.

[8] Lombard J-EW, Moxey D, Sherwin SJ, Hoessler JF, Dhandapani S, Taylor MJ Implicit large-eddy simulation of a wingtip vortex. AIAA J 2015;54(2):506-18.

[9] Kerkemeier S, Parker S, Fischer P. scalability of the nek5000 spectral element code. Technical Report. Jülich blue gene/p extreme scaling workshop; 2010.

[10] Abide S, Binous MS, Zeghmati B. An efficient parallel high-order compact scheme for the $3 \mathrm{~d}$ incompressible Navier-Stokes equations. Int J Comut Fluid Dyn 2017;31(4-5):214-29.

[11] Gellert M, Rüdiger G. Stratorotational instability in Taylor-Couette flow heated from above. J Fluid Mech 2009;623:375-85.

[12] Hide R. An experimental study of thermal convection in a rotating liquid. Phil Trans R Soc Lond A 1958;250(983):441-78.

[13] Randriamampianina A, Crespo del Arco E. Inertia-gravity waves in a liquidfilled, differentially heated, rotating annulus. J Fluid Mech 2015;782:144-77.

[14] Schornbaum F, Rude U. Massively parallel algorithms for the lattice Boltzmann method on nonuniform grids. SIAM J Sci Comput 2016;38(2):C96-C126.

[15] Vàzquez M, Houzeaux G, Koric S, Artigues A, Aguado-Sierra J, Ars R, et al. Alya: multiphysics engineering simulation toward exascale. J Comput Sci 2016;14:15-27.

[16] Baker AH, Falgout RD, Gamblin T, Kolev TV, Schulz M, Yang UM. Scaling algebraic multigrid solvers: on the road to exascale. In: Competence in high performance computing. Springer; 2011. p. 215-26.

[17] Notay Y. An aggregation-based algebraic multigrid method. Electron Trans Numer Anal 2010;37(6):123-46.

[18] Laizet S, Lamballais E, Vassilicos J. A numerical strategy to combine high-order schemes, complex geometry and parallel computing for high resolution DNS of fractal generated turbulence. Comput Fluids 2010;39(3):471-84.

[19] He P. A high order finite difference solver for massively parallel simulations of stably stratified turbulent channel flows. Comput Fluids 2016;127:161-73. doi:10.1016/j.compfluid.2015.12.012.

[20] Thais L, Tejada-Martnez AE, Gatski TB, Mompean G. A massively parallel hybrid scheme for direct numerical simulation of turbulent viscoelastic channel flow. Computers \& Fluids 2011;43(1):134-42.

[21] Chatterjee AG, Verma MK, Kumar A, Samtaney R, Hadri B, Khurram R. Scaling of a fast fourier transform and a pseudo-spectral fluid solver up to 196608 cores. J Parallel Distrib Comput 2018:113:77-91.

[22] Shi L, Rampp M, Hof B, Avila M. A hybrid mpi-openmp parallel implementation for pseudospectral simulations with application to taylorcouette flow. Comput Fluids 2015;106:1-11.
[23] Mininni PD, Rosenberg D, Reddy R, Pouquet A. A hybrid MPI-Openmp scheme for scalable parallel pseudospectral computations for fluid turbulence. Paralle Comput 2011;37(6):316-26.

[24] Lele SK. Compact finite difference schemes with spectral-like resolution. Comput Phys 1992;103(1):16-42.

[25] Sun X-H. Application and accuracy of the parallel diagonal dominant algorithm. Parallel Comput 1995;21(8):1241-67.

[26] Haldenwang P, Labrosse G, Abboudi S, Deville M. Chebyshev 3d spectral and $2 \mathrm{~d}$ pseudospectral solvers for the Helmholtz equation. J Comput Phys $1984 ; 55: 115-28$

[27] Hugues S, Randriamampianina A. An improved projection scheme applied to pseudospectral methods for the incompressible Navier-Stokes equations. Int Numer Methods Fluids 1998;28:501-21.

[28] Le Bars M, Le Gal P. Experimental analysis of the stratorotational instability in a cylindrical Couette flow. Phys Rev Lett 2007;99(6):064502.

[29] Rüdiger G, Hollerbach R, Kitchatinov LL. Magnetic processes in astrophysics: theory, simulations, experiments. John Wiley \& Sons; 2013.

[30] Ibanez R, Swinney HL, Rodenborn B. Observations of the stratorotational instability in rotating concentric cylinders. Phys Rev Fluids 2016;1:053601.

[31] Rüdiger G, Seelig T, Schultz M, Gellert M, Egbers C, Harlander U. The stratorotational instability of Taylor-Couette flows with moderate Reynolds numbers. Geophys Astrophys Fluid Dyn 2017;111(6):429-47.

[32] Pierrehumbert R, Swanson K. Baroclinic instability. Annu Rev Fluid Mech 1995;27(1):419-67.

[33] Orlanski I, Cox MD. Baroclinic instability in ocean currents. Geophys Astrophys Fluid Dyn 1972;4(1):297-332.

[34] Read PL, Collins M, Früh W-G, Lewis SR, Lovegrove AF. Wave interactions and baroclinic chaos: a paradigm for long timescale variability in planetary atmospheres. Chaos Solitons Fractals 1998;9(1-2):231-49.

[35] Read PL. Transition to geostrophic turbulence in the laboratory, and as a paradigm in atmospheres and oceans. Surv Geophys 2001:22(3):265-317.

[36] Fritts DC, Alexander MJ. Gravity wave dynamics and effects in the middle atmosphere. Rev Geophys 2003;41(1).

[37] Borchert S, Achatz U, Fruman MD. Gravity wave emission in an atmospherelike configuration of the differentially heated rotating annulus experiment. J Fluid Mech 2014;758:287-311

[38] Hien S, Rolland J, Borchert S, Schoon L, Zülicke C, Achatz U. Spontaneous inertia-gravity wave emission in the differentially heated rotating annulus experiment. J Fluid Mech 2018;838:5-41.

[39] Von Larcher T, Viazzo S, Harlander U, Vincze M, Randriamampianina A. Instabilities and small-scale waves within the Stewartson layers of a thermally driven rotating annulus. J Fluid Mech 2018:841:380-407.

[40] Raspo I, Hugues S, Serre E, Randriamampianina A, Bontoux P. A spectral projection method for the simulation of complex three - dimensional rotating flows. Comput Fluids 2002;31(4-7):745-67.

[41] Abide S, Viazzo S. A 2d compact fourth-order projection decomposition method. J Comput Phys 2005;206(1):252-76.

[42] Knikker R. Study of a staggered fourth-order compact scheme for unsteady incompressible viscous flows. Int I Numer Methods Fluids 2009·59(10):1063-92.

[43] Schiestel R, Viazzo S. A hermitian-fourier numerical method for solving the incompressible Navier-Stokes equations. Comput fluids 1995;24(6):739-52.

[44] Abide S, Chesneau X, Zeghmati B. A fourth-order iterative solver for the singular poisson equation. In: Dimov I, Faragó I, Vulkov L, editors. Numerical analysis and its applications. Berlin, Heidelberg: Springer Berlin Heidelberg; 2013. p. 143-50. ISBN 978-3-642-41515-9.

[45] Pekurovsky D. P3DFFT: a framework for parallel computations of fourier transforms in three dimensions. SIAM J Sci Comput 2012;34(4):C192-209.

[46] Li N, Laizet S. 2DECOMP\&FFT-a highly scalable 2d decomposition library and FFT interface. In: Proceedings of the Cray User Group conference; 2010. p. 1-13.

[47] Roache PJ. Code verification by the method of manufactured solutions. J Fluids Eng 2002;124(1):4-10

[48] Harlander U, von Larcher T, Wang Y, Egbers C. PIV- and LDV-measurements of baroclinic wave interactions in a thermally driven rotating annulus. Exp Fluids 2011;51(1):37-49.

[49] von Larcher T, Dörnbrack A. Numerical simulations of baroclinic driven flows in a thermally driven rotating annulus using the immersed boundary method. Meteorol Z 2014:1-12

[50] Vincze M, Borchert S, Achatz U, von Larcher T, Baumann M, Liersch C, et al. Benchmarking in a rotating annulus: a comparative experimental and numerical study of baroclinic wave dynamics. Meteorol Z 2015;23(6):611-35. 